\title{
GÉOGRAPHIE, TYPOLOGIE, POLITIQUE : \\ LA FONDATION DE LA CHAIRE DES LANGUES FINNO-OUGRIENNES \\ À L'ÉCOLE DES LANGUES ORIENTALES VIVANTES
}

\author{
ILDIKÓ JÓZAN
}

\begin{abstract}
Département de littérature et culture comparées, Université ELTE de Budapest jozan.ildiko@btk.elte.hu
\end{abstract}

\begin{abstract}
L'article retrace les débuts de la carrière d'Aurélien Sauvageot, " l'homme de France qui connaît le mieux hommes et choses de Hongrie ». Elève d'Antoine Meillet, figure-clé des relations culturelles franco-hongroises de l'entre-deux-guerres, premier titulaire, en 1931, de la Chaire des langues finno-ougriennes à l'Ecole des langues orientales à Paris, Sauvageot fait son entrée sur la scène scientifique, littéraire et politique française et hongroise à la sortie de la première guerre mondiale dans laquelle la Hongrie et la France étaient dans les camps opposés. L'arrivée de Sauvageot à Budapest (en 1923) et ses débuts dans le milieu de la linguistique finno-ougrienne permettaient de croire, pour la science et la politique françaises, que ce jeune linguiste bientôt hungarophone serait capable de servir la propagande intellectuelle, c'est-à-dire « la cause » française en Hongrie. Dans le sens inverse, aux yeux des autorités hongroises, on espérait qu'il pût disséminer la propagande intellectuelle hongroise en France. Or les objectifs communs (le rapprochement intellectuel) ne pouvaient compenser les contradictoires entre les deux pays, ces dernière se sont constamment imposées sur le chemin d'Aurélien Sauvageot.
\end{abstract}

Mots-clés : Aurélien Sauvageot, Antoine Meillet, langue hongroise, École nationale des langues orientales, Collège Eötvös, enseignement du hongrois à Paris, relations politiques et culturelles franco-hongroises, entre-deux-guerres

Le 6 décembre 1929, Louis de Vienne, ministre de France en Hongrie, adresse une lettre « à son excellence Monsieur Aristide Briand, ministre des Affaires étrangères $»$ :

D'après des renseignements qui me sont communiqués et qui auraient été recueillis par M. Paul Boyer, directeur de l'Ecole des Langues Orientales et par M. Meillet, professeur au Collège de France, la création d'une chaire de hongrois et de finnois à l'École des Langues Orientales serait de nouveau envisagée, mais, cette fois, au titre exclusivement français et par attribution de crédits sur le budget de notre ministère de l'Instruction Publique. Cet enseignement pourrait commencer à partir du mois de novembre 1930. [...] 
Je ne puis que souhaiter vivement la réalisation de ce projet. Cela serait du meilleur effet sur l'opinion publique de ce pays devant laquelle ennemis ou même "amis", car il en est parmi ceux-ci qui ont aussi intérêt à nous nuire, établissent volontiers des comparaisons à notre détriment entre l'estime en laquelle certains, notamment les Allemands et les Italiens, tiennent la culture hongroise et le dédain que nous lui marquons. Ce geste de notre part aurait des conséquences que le sacrifice pécuniaire modique à consentir paierait à fort bon marché. Le Hongrois est très sensible à la politesse. Flatté de son amour-propre, il se tournerait d'avantage encore vers l'étude de notre langue; il se montrerait encore plus désireux de notre enseignement. Or l'attrait que l'on éprouve ici pour la culture française est peut-être notre seul et en tout cas notre meilleur instrument. [...]

J'ajoute qu'à mon sentiment cette chaire devrait être attribuée à $\mathrm{M}$. Sauvageot. Comme le sait le Département, cet universitaire, actuellement détaché à Budapest, est un spécialiste des langues finno-ougriennes. Il a eu récemment une soutenance de thèse particulièrement remarquée. Au surplus, sa finesse d'esprit, sa puissance de travail et l'aisance de son élocution le mettent au premier rang.

Dans un autre ordre d'idées, le départ éventuel de M. Sauvageot nous permettrait de concentrer notre effort pécuniaire et de réunir sur une seule personne des crédits nous permettant d'avoir à Budapest, sinon en droit, du moins en fait, cet « attaché intellectuel » dont le besoin se fait de plus en plus sentir. ${ }^{1}$

Ce document est loin d'être le seul, ni le premier, ni même le dernier ou le plus décisif de ceux qui ont été produits au sujet de la fondation de la chaire des langues finno-ougriennes ${ }^{2}$ à l'École des langues orientales vivantes. Mais il est particulièrement intéressant, car il marque l'aboutissement d'un processus et d'un projet dont les principales lignes ont été tracées sur un tableau différent de l'Europe, avant la guerre ; son enjeu principal est la mise en valeur en France de la langue et de la culture hongroises, bien que la Hongrie ait été placée, pour son action politique, sur le banc des principaux accusés du conflit mondial. On peut lire ce document comme une lettre de congé par laquelle Louis de Vienne remet la question dans les mains des autorités françaises en la retirant du même coup de celles de ses partenaires hongrois. Il met fin aux négociations entre la France et la Hongrie dont, depuis la fin de la guerre, le ministre de France en Hongrie (lui-même en personne et ses prédécesseurs) était un acteur important. Plus personnellement, il clôt une affaire qui, dès 1925 et à plusieurs reprises, a failli lui échapper des mains.

Sur le terrain politique, la question de l'enseignement du hongrois à l'École des langues orientales vivantes est revenue à l'ordre du jour avec la reprise, après la guerre, des relations intellectuelles entre la France et la Hongrie. La langue hongroise était déjà enseignée à Paris, depuis 1908, grâce aux cours assurés par Ignác Kont jusqu’à son décès en décembre 1912, puis, en 1913 et 1914, par Louis 
Eisenmann avec Joseph Fóti à ses côtés en tant que répétiteur. ${ }^{3}$ Dès la fin de l'année 1909, le gouvernement hongrois avait proposé d'assurer, à part égale avec le ministère français, la subvention « d'un enseignement régulier de hongrois qui serait confié à M. Kont $»^{4}$, mais la proposition n'aboutit pas et la guerre inscrivit une césure dans l'histoire institutionnelle des relations intellectuelles franco-hongroises, jusqu'à ce qu'en octobre 1920, Louis Eisenmann, chargé par le ministère des Affaires étrangères « de prendre le contact avec le Gouvernement hongrois en vue d'établir des relations intellectuelles entre les deux pays », se rende à Budapest. À son retour, ce dernier remet aux ministères de l'Instruction publique et des Affaires étrangères « un aide-mémoire [...] où il envisage les modalités que pourraient prendre ces relations dans les différents degrés de l'enseignement primaire, secondaire et supérieur $»$. En ce qui concerne le supérieur, il propose, entre autres, à des universités hongroises la création de « chaires françaises, occupées par des Français ». Pour ce qui est de la « chaire hongroise de l'Université de Paris », il note : « Suppression, maintien, transformation. La chaire ne peut être occupée que par un Français », puis il ajoute : " création à la Sorbonne d'un lectorat, confié à un Hongrois, pour l'enseignement de la langue » et, à l'École des langues orientales vivantes, « chaire, cours complémentaires, lectorat $»^{5}$. Du point de vue pratique, le document n'évoque pas le problème majeur qui se pose du côté français : il n'existe pas un seul spécialiste français qui soit capable d'assumer cette tâche.

Au demeurant, si les hostilités ont suspendu la coopération intellectuelle et les relations universitaires officielles entre la France et la Hongrie, la période de la guerre est loin d'être négligeable dans l'histoire de la création de la chaire des langues finno-ougriennes à l'École des langues orientales vivantes. La volonté de trouver un spécialiste français pour l'étude des langues finno-ougriennes (seules langues en Europe qui ne font pas partie des langues indo-européennes), et notamment pour le hongrois, s'imposait de manière de plus en plus pressante, tout d'abord en raison même de l'évolution qu'avait connu la linguistique en tant que discipline depuis le début du siècle, mais aussi du besoin immédiat, en temps de guerre, d'information dans les langues de l'Autriche-Hongrie, et enfin des ravages de la guerre dans les rangs des linguistes. La figure-clé qui, aux côtés de l'administrateur de l'École, Paul Boyer, anima, sur le terrain non seulement scientifique, mais aussi politique, toute cette histoire du début jusqu'à la fondation est Antoine Meillet, auquel la lettre du ministre de France en Hongrie fait référence avec son titre de " professeur au Collège de France ». De fait, en examinant de plus près son rôle, on se rend compte que celui-ci dépasse de loin les cadres que ce titre laisserait supposer.

Qui est Meillet et en quel sens joue-t-il le rôle principal dans la fondation de la chaire des langues finno-ougriennes à l'École des langues orientales, et plus encore dans les études du hongrois en France ? On peut ajouter : et dans la formation de l'image de la langue hongroise en France entre les deux guerres? 
Antoine Meillet (1866-1936) étudie à la Sorbonne à partir de 1885 et fréquente en même temps les cours de Michel Bréal au Collège de France et de Ferdinand de Saussure et Sylvain Lévi à l'École pratique des hautes études. «En 1890, il se rend en mission durant un an dans le Caucase pour étudier l'arménien moderne et dès son retour, il est appelé à remplacer Saussure en grammaire comparée, cours qu'il complète à partir de 1894 par une conférence en iranien. [...] Il obtient en 1902 la chaire d'arménien de l'École des Langues orientales et succède à Bréal en 1906 au Collège de France où il poursuit un enseignement de linguistique consacré à l'histoire et à la structure des langues indo-européennes, parallèlement aux formations qu'il dispense à l'École pratique des hautes études. [...] À partir de 1906, Meillet marque son intérêt pour la définition de lois générales, concernant l'ensemble de la linguistique $»^{6}$. Il se penche notamment sur les relations entre les différentes langues et types de langues et sur les faits de civilisation que la formation des langues permet d'observer. Il gagne une autorité importante parmi les linguistes et dans la vie intellectuelle en général. «Parmi ses activités, à la frontière du politique et des langues, il est appelé comme expert pour contribuer à la définition des groupes, des langues et des frontières au moment des traités consécutifs à la guerre de 14-18 $»^{7}$. À cette époque, « Meillet rédigea des livres où il s'intéresse aux liens entre langues, peuples et nations : les Caractères généraux des langues germaniques (1917) et, surtout, Les langues dans l'Europe nouvelle (1918) ; ce dernier ouvrage, dont Meillet fit paraître une deuxième édition en 1928, augmentée de données statistiques rassemblées par son disciple Lucien Tesnière $»^{8}$, avait pour but d'exposer, selon l'auteur, «la situation linguistique de l'Europe telle qu'elle est, et non comme les vanités et les prétentions nationales exaspérées depuis le XIX ${ }^{\mathrm{e}}$ siècle souhaitaient qu'elle soit »".

Dès qu'il commence à enseigner, Meillet encourage les recherches sur les langues finno-ougriennes qui l'intéressent dans l'optique d'une comparaison avec les langues indo-européennes, d'autant plus que cette branche de la linguistique n'a pas de spécialiste en France et qu'elle se sert surtout de l'allemand pour ses communications ${ }^{10}$. Un de ses étudiants, Robert Gauthiot, commence à s'en occuper, il apprend « à utiliser, avec une véritable aisance, tout le groupe des langues finno-ougriennes » et publie deux articles (en 1908 et en 1910) qui «montrent le parti que savait tirer Gauthiot de l'indo-européen pour éclairer les faits finno-ougriens ${ }^{11}$, mais il décède en septembre 1916 des suites d'une blessure de guerre. C'est ainsi qu'en 1918, Meillet fait appel à un lycéen, Aurélien Sauvageot (1897-1988), âgé d'à peine vingt ans, mais qui assiste à ses cours depuis déjà deux ans. ${ }^{12}$

Sauvageot - comme il le dit lui-même dans ses mémoires écrites et publiées soixante-dix ans après les événements - " prépare le concours d'admission à l'École normale supérieure. Il veut entrer à l'université, se consacrer à l'étude des langues. Il s'est déjà spécialisé dans les langues germaniques : anglais, allemand 
et langues scandinaves. Il s'est lancé à corps perdu dans cette étude $»^{13}$. Pourtant Meillet le destine à une autre carrière et lui prépare un tout autre avenir. Avec Paul Boyer et Joseph Vendryes (professeur à la Sorbonne), ils se mettent d'accord pour constater que seul Sauvageot est capable de prendre la succession de Gauthiot et d'introduire l'étude des langues finno-ougriennes en France. ${ }^{14}$ " Vous êtes né à Constantinople ; vous avez parlé le turc osmanli. Cette langue a une structure qui ressemble beaucoup à celle des langues finno-ougriennes. Et puis, vous savez bien le suédois, par lequel il vous faudra passer pour apprendre le finnois, et comme vous êtes aussi un bon allémaniste, vous aurez facilement accès au hongrois »-dit Meillet à son élève. ${ }^{15}$

Sauvageot passe son bac ; en avril 1918, il est admis à l'École normale supérieure et le projet se met en place. En octobre, il part pour la Suède, puis en juin 1919 pour la Finlande, d'où il rentre en octobre pour reprendre ses cours à Paris. Du $1^{\text {er }}$ décembre 1919 jusqu'en octobre 1923, il est « détaché au ministère des Affaires étrangères $»^{16}$ ( " attaché comme rédacteur-interprète au Bureau d'étude de la presse étrangère, pour la presse suédoise et finlandaise $\gg^{17}$ ) et il s'inscrit parallèlement en 1919-1920 et 1920-1921 à l'Ecole des langues orientales pour apprendre le russe et le turc. En 1921 et 1922, il fait plusieurs missions en Allemagne ; en 1923, il se met à l'épreuve d'une agrégation d'allemand - sans succès ${ }^{18}$. En novembre de la même année, il part pour Budapest, dans l'objectif d'enseigner le français au Collège Eötvös (l'Ecole normale de Budapest) et, comme il le souligne dans ses mémoires, " pour apprendre la langue, écrire mes deux thèses de doctorat et me qualifier aussi complètement que possible pour occuper la chaire de langues finno-ougriennes que mes patrons parisiens s'évertuaient à faire fonder à l'École nationale des langues orientales ${ }^{19}$.

À l'âge de 20 ans, au moment où Meillet lui a fait part de la carrière qu'il a imaginée pour lui, Sauvageot ne savait rien des langues finno-ougriennes et n'en parlait encore aucune. À 26 ans, en novembre 1923, lorsqu'il part pour Budapest avec la promesse de retrouver quelques années plus tard, à son retour à Paris, un poste d'enseignant à l'École, le voyage l'inquiète, le pays lui est inconnu et il ne parle toujours pas un mot de hongrois..$^{20}$ Dans une lettre adressée avant son départ de Paris, le 29 octobre 1923, au directeur du Collège Eötvös, Géza Bartoniek, il écrit :

Depuis des années, je nourris le désir de me consacrer entièrement aux études finno-ougriennes dans lesquelles je ne suis encore, hélas, que très novice. Des déboires universitaires m'ont empêché de réaliser mon projet dès l'an dernier. Vous me trouverez donc animé de la plus vive intention et du plus ferme propos de rattraper le temps perdu. [...]

Je m'excuse d'ailleurs auprès de vous, Monsieur le Directeur, de ne savoir encore presque rien de votre langue et de votre littérature. Je ne lis le magyar que fort difficilement, car si sa structure grammaticale 
m'est familière, son vocabulaire me fait défaut et son usage me manque complètement. Il me reste l'espoir de remplir au plus tôt cette immense lacune. Je crois pouvoir vous promettre que j'y mettrai toutes mes forces et toute ma volonté. ${ }^{21}$

En effet, il ne tarde pas à pouvoir être présenté, en Hongrie et en France, comme un spécialiste des langues finno-ougriennes. Dès 1924, dans le numéro d'avril-septembre de la Revue des Études hongroises et finno-ougriennes, il publie « un court exposé de l'état où en est actuellement la question des origines hongroises », article qui est le résultat de sa polémique avec Étienne Zichy. Ce dernier venait de résumer dans un premier article en français sa réflexion sur le sujet $^{22}$ mais, comme l'indique une note de la rédaction, " pris de scrupules » il s'est refusé « très amicalement à livrer à nos lecteurs la suite de son manuscrit » et il a « laissé à notre confrère $\mathrm{A}$. Sauvageot le soin de terminer son article $»^{23}$. Précisons que Sauvageot intervient dans le cadre du débat sur les thèses de Zichy publiées en 1922 au sujet de l'origine du peuple hongrois, débat qui a lieu en hongrois au sein des milieux scientifiques hongrois et auquel participent $\mathrm{Zi}$ chy lui-même, bien sûr, mais aussi d'autres éminents linguistes (en premier lieu Gyula Németh et Zoltán Gombocz). ${ }^{24}$ Or, en dépit du caractère bien hongrois de cette polémique (toujours en cours), l'auteur et la revue ont publié en français plusieurs articles sur la question. En laissant aux linguistes le soin de mesurer l'apport personnel de Sauvageot à ladite polémique, nous nous contenterons de souligner que l'image qu'il dessine des Hongrois est celle d'un peuple supérieur aux autres peuples finno-ougriens. Pour lui, les Hongrois

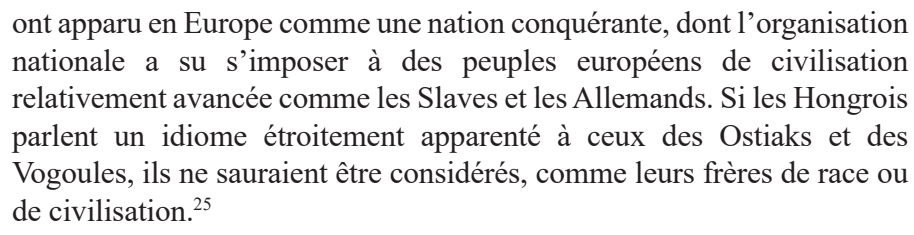

Or, quelques pages plus loin, il ajoute, sous forme de question : «Mais pour être un peuple de conquérants est-il besoin d'avoir accédé à un niveau supérieur de civilisation ? $\|{ }^{26} \mathrm{~S}$ 'il ne donne pas de réponse, le fait même de poser la question semble pouvoir signifier que la réponse est plutôt négative.

À en croire une lettre que le "maître" hongrois, Zoltán Gombocz, adresse à Meillet le 25 mai 1924, " par ses qualités personnelles et par son érudition très sérieuse » Sauvageot a gagné « dès les premières semaines de son séjour à Budapest [...], la sympathie et la considération des linguistes hongrois $»^{27}$. Si, malgré tout, on peut douter de l'entière franchise ou plutôt de la gratuité de ces propos, ce n'est pas seulement à cause de Sauvageot, ${ }^{28}$ mais aussi en raison du contexte 
politique et intellectuel de l'époque. L'arrivée de Sauvageot à Budapest et ses débuts sur la scène de la linguistique finno-ougrienne permettaient, tant du point de vue scientifique et politique et à la fois du côté français et hongrois, de croire que le linguiste, en tant que français hungarophone, pourrait servir respectivement « la cause française » - la propagande intellectuelle française en Hongrie devant un public hongrois - ou, à l'inverse, la propagande intellectuelle hongroise en France. C'est certainement grâce à cet intérêt commun que Gombocz écrit à Meillet et Meillet, à son tour, au Service des Euvres du ministère français des Affaires étrangères pour défendre le poste de Sauvageot quand, au printemps 1924, il est question, pour la première fois et non la dernière, de rappeler le jeune lecteur en France. Malheureusement, on ne peut s'appuyer que sur les Souvenirs de Sauvageot lui-même pour se renseigner sur la raison de cette menace. En l'occurence, elle aurait été envisagée à la suite à d'une conférence tenue en allemand, en mars 1924, devant la Société linguistique hongroise, l'allemand et l'influence intellectuelle allemande étant à cette époque le principal adversaire pour la propagande française en Hongrie.

Le 21 septembre 1924, « en sachant de quel mérite [il jouit] aux yeux de Meillet », Sauvageot adresse à son tour une lettre à Zoltán Gombocz où il lui demande d'intervenir en sa faveur auprès de son collègue français afin que lui soit octroyé un délai supplémentaire pour la remise de ses thèses sur les rapports ouralo-altaïques (thèse principale) et sur le poète hongrois, Endre Ady (thèse complémentaire ${ }^{29}$. Meillet aurait voulu qu'elles soient présentées en avril 1925 , or Sauvageot ne se sent « nullement à la hauteur de ces deux tâches »; il explique « les raisons qui ont poussé Meillet à [le] hâter d'une manière aussi pressante » :

\begin{abstract}
Si M. Meillet est si pressé de me voir docteur de l'université de Paris, c'est qu'il pense pouvoir m'appuyer à ce moment avec plus de facilité pour l'obtention d'une chaire de langue et littérature hongroises à l'École des langues orientales de Paris. Cette chaire, il faut la fonder et pour cela, il convient que le candidat proposé se soit acquis le plus possible de mérites. Telle est la situation.

De mon côté, je ne dissimule pas que je suis très peu préparé à enseigner le hongrois. J'estime que deux années de séjour en Hongrie me seraient indispensables, et cela dans l'hypothèse où je pourrais m'adonner librement à l'étude de la langue, sans être harassé par un travail pressé et aussi absorbant que celui qui consiste à rédiger une thèse de doctorat d'une pareille envergure. ${ }^{30}$
\end{abstract}

La même année, Sauvageot signe un article sur les « Langues finno-ougriennes et langues samoyèdes » dans un ouvrage intitulé Les Langues du monde, écrit " par un groupe de linguistes sous la direction d'Antoine Meillet et Marcel Cohen ». Les différents chapitres du livre analysent les situations linguistiques respectives de chacune des langues et de chacune des familles de langue sous 
un double aspect : du point de vue de l'évolution linguistique et celui de la « civilisation » qu'elles représentent; l'ouvrage vise donc « à donner une idée de la répartition des langues dans le monde, en tenant compte de leur histoire $\|^{31}$. Dans la deuxième partie de son article, intitulé « Les langues de civilisation », Sauvageot écrit :

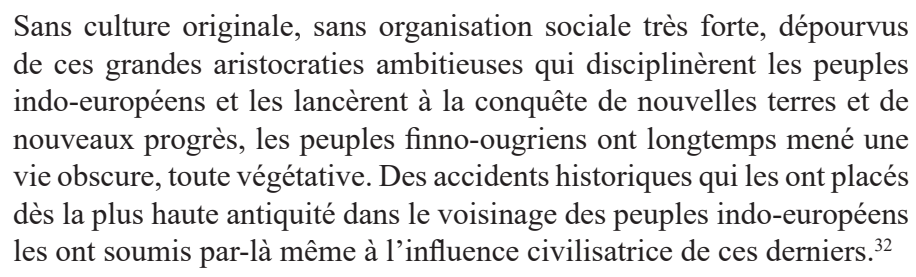

Un peu plus loin, il ajoute :

Conservant leurs éléments primitifs, les langues finno-ougriennes de civilisation ont changé totalement de caractère sous l'influence des langues indo-européennes de civilisation. Elles n'en seront bientôt plus que des décalques dont seuls les matériaux rappelleront leur origine et leur continuité historiques. ${ }^{33}$

Les langues finno-ougriennes sont donc présentées dans le cadre d'une comparaison avec les peuples de langues indo-européennes, que Sauvageot décrit comme étant plus avancés, plus développés, disposant d'une culture originale. Dans sa conception, c'est à ces derniers que revient indirectement le mérite d'avoir contribué à l'évolution de la civilisation finno-ougrienne.

Il semble que ces propos s'inscrivent dans le cadres qu'Antoine Meillet a donné à la réflexion sur la situation linguistique de l'Europe dans une série de publications apparues depuis les années de guerre. Dans ces études, Meillet prend pour point de départ certains faits historiques, d'ordre linguistique ou général, dans le but de mesurer, dans un deuxième temps, la valeur d'une civilisation dans le monde contemporaine, l'unité de mesure étant invariablement les langues et les civilisations indoeuropéennes. Selon Meillet, auteur des Langues dans l'Europe nouvelle (première édition en 1918, seconde, revue et corrigée dans le nouveau contexte politico-historique, en 1928), « le monde tend à n'avoir qu'une civilisation » ainsi que « toutes les langues de l'Europe tendent à être, par ce qu'elles expriment, le calque fidèle les unes des autres $»^{34}$.

En 1915, dans son article intitulé Les Langues et les nationalités, Meillet déclare : "Chaque nation slave s'est donné au cours du XIX ${ }^{\mathrm{e}}$ siècle, une langue de civilisation qui répond à ses besoins $»^{35}$. En 1918, lorsqu'il compare, dans la même revue, « la Situation linguistique en Russie et en Autriche-Hongrie », il souligne qu'en Russie, « on rencontre des populations de langues diverses » mais 
le russe est la seule langue de civilisation de l'immense majorité, et souvent de l'unanimité, de la population. Le russe a eu, au cours du $\mathrm{XIX}^{\mathrm{e}}$ siècle, l'une des littératures des plus originales, des plus humaines. Et il a été assoupli de manière à rendre aisément toutes les idées philosophiques, toutes les notions scientifiques. C'est une grande langue de civilisation, moins connue que le français, l'italien, l'allemand ou l'anglais, mais qui peut rendre les mêmes services. ${ }^{36}$

En revanche, les langues de l'Autriche-Hongrie, « réunis par le hasard [...], aspirent à se dissocier » et « se refusent à accepter pour langue officielle et pour langue de civilisation le magyar ou l'allemand ». Le magyar est « sans aucun rapport linguistique, soit avec les langues slaves, $[\ldots]$ soit avec une langue romane [...]. Par son type, il est éloigné des autres langues de l'Europe qui appartiennent presque toutes au groupe indo-européen $»^{37}$. En 1918, dans Les Langues dans l'Europe nouvelle, il ajoute :

\begin{abstract}
Du reste, le magyar n'est pas une vieille langue de civilisation. Il porte dans son vocabulaire la trace d'influences extérieures de toutes sortes; il est plein d'emprunts au turc, au slave, à l'allemand, au latin, tandis que lui-même n'a exercé sur les langues voisines presque aucune influence durable. Sa littérature n'a pas de prestige. Il n'appartient pas à la même famille linguistique que la plupart des langues parlées en Europe et que toutes celles qui se parlent en Autriche-Hongrie ; il a une structure compliquée, n'est facile à apprendre pour personne. Hors de la Hongrie, il est universellement inconnu. Sorti des frontières de la Hongrie, un sujet hongrois qui ne sait pas d'autre langue commune est hors d'état de se faire entendre, hors d'état même presque partout de trouver un interprète. Une publication scientifique en magyar est destinée à demeurer ignorée. ${ }^{38}$
\end{abstract}

Ces passages réapparaissent dans l'édition de 1928 avec un petit ajustement géopolitique (« et que toutes celles qui se parlent en Autriche-Hongrie » est remplacé par " et surtout dans cette région de l'Europe ») et avec un rajout dans la dernière phrase qui adoucit ses propos sur les publications scientifiques («[...] en magyar, quelle qu'en soit la valeur, est $[\ldots] \gg) .{ }^{39}$

Pour revenir à la lettre de Louis de Vienne de décembre 1929, ce n'est pas en 1930 que l'on a pu assister au premier cours de Sauvageot inaugurant la chaire des langues finno-ougriennes à l'École des langues orientales mais un an plus tard, en novembre 1931. De 1923 à 1931, Sauvageot a donc passé huit ans à Budapest en travaillant beaucoup. Il s'investit à fond dans l'étude de la langue hongroise et des langues finno-ougriennes, il fait surtout des recherches sur la formation (la préhistoire ou les origines) des langues. En deux ans seulement, il apprend remarquablement bien le hongrois puis il continue à progresser jusqu'à « la perfection » que saluent régulièrement ses contemporains hongrois. Il commence 
à traduire, il enseigne au Collège Eötvös et, plus tard, il donne également des cours à l'Université de Budapest. Une fois sa thèse achevée qu'il soutient finalement en juin 1929 (à la Sorbonne) ${ }^{40}$, il entreprend de rédiger un grand dictionnaire franco-hongrois. La presse hongroise le salue à cette occasion en soulignant qu'en la personne de Sauvageot, le grade de docteur est pour la première fois accordé pour distinguer la connaissance de la langue et la culture hongroises ${ }^{41}$. Un peu plus tard, Paul Boyer écrit dans le document qui propose sa promotion au poste de professeur délégué à l'École des langues orientales : « De l'avis de tous ceux qui le connaissent pour avoir été ses maîtres ou l'avoir suivi en son activité de linguiste, MM. A. Meillet, Marcel Cohen, moi-même, M. Sauvageot, par sa science et par ses aptitudes à l'enseignement oral, fera honneur à l'Ecole des langues orientales ». Et il ajoute : « Il est aujourd'hui l'homme de France qui connait le mieux hommes et choses de Hongrie ${ }^{42}$. En effet, il restera encore longtemps le seul spécialiste français du hongrois et de la culture hongroise, peut-être pas unanimement reconnu par tous, ni en France, ni en Hongrie, mais au moins prêt à se mettre au service des deux causes.

Lors de son séjour à Budapest, de son propre gré, il a déjà officieusement ou officiellement proposé ses services aux autorités françaises et hongroises. Au printemps 1925, il s'engage « sans aucun mandat $»^{43}$ dans des discussions avec le gouvernement hongrois sur des projets destinés à « resserrer les liens intellectuels qui unissent la France et la Hongrie $»^{44}$. La fondation à Paris d'une chaire de langue hongroise à l'Ecole des langues orientales (un professeur et un lecteur) en fait partie, avec la participation du gouvernement hongrois aux dépenses. Fin mai de la même année, il accompagne à Paris Zoltán Magyary, chef de service du ministère de l'Instruction publique, qui s'y rend « avec la mission de travailler au rapprochement intellectuel » entre la France et la Hongrie ${ }^{45}$. Ils rencontrent ensemble Paul Boyer ainsi que, au ministère des Affaires étrangères, Jean Marx, avec lesquels il évoquent, entre autres, le sujet de la chaire. Suite à ces visites, le ministère des Affaires étrangères fait savoir au ministre français de l'Instruction publique que vu « l'intérêt spécial que présenterait la création d'une chaire de langue et de littérature hongroises » à l'École et «pour faciliter la réalisation de ce projet », il est prêt à mettre à sa disposition « pour le dernier trimestre de l'année courante, une somme nécessaire aux indemnités à verser au titulaire de la chaire et au répétiteur qui lui serait adjoint $»^{46}$. Mais le projet fait long feu, entre autres faute de candidat au poste de professeur qui puisse convenir aux deux partenaires.

Quant à Sauvageot, Meillet ${ }^{47}$ et Jean Marx lui signalent qu'il a outrepassé le cadre de ses activités et le rappellent à son devoir. Marx lui adresse une lettre amicale mais très ferme : 


\begin{abstract}
Mon cher Ami,
Vous savez l'amitié que je vous porte et l'intérêt avec lequel je suis vos travaux. C'est pourquoi je tiens à préciser pour vous les conditions dans lesquelles va s'ouvrir l'année prochaine en Hongrie. [...] il s'en est fallu de peu, à certains moments, que votre rappel ne fût demandé, ce qui nous aurait mis, sans aucun doute, dans l'obligation de l'accorder. [...] Votre mission en Hongrie doit consister, semble-t-il, avant tout dans des travaux d'ordre purement scientifique et linguistique, plutôt que dans une action d'expansion intellectuelle que nous ne vous demandons pas et que la Légation n'a pas l'intention de vous confier. Je vous prie instamment de croire qu'en vous donnant ces conseils et en vous indiquant avec franchise et netteté la situation, j'ai conscience de servir de la manière la plus utile vos intérêts et votre carrière. ${ }^{48}$
\end{abstract}

À la fin de 1925, c'est pourtant le ministre de France en Hongrie lui-même qui fait appel à Sauvageot pour lui demander de corriger une traduction maladroite du ministère hongrois lors de l'affaire des faux billets français. Le linguiste obtempère et, de cette manière, il rend aussi un grand service au gouvernement hongrois ${ }^{49}$. Les demandes officielles de traduction de la part de la Légation deviennent bientôt régulières ${ }^{50}$, mais elles ne donnent pas lieu à l'octroi en bonne et due forme d'une mission officielle pour le rapprochement intellectuel franco-hongrois.

Quoi qu'il en soit, il semble que Sauvageot n'a pas retenu ou n'a pas été dans l'obligation de retenir longtemps "le conseil amical" de Marx. De fait, il était, à Budapest, le mieux informé des intentions du gouvernement hongrois, mieux encore que la Légation de France. C'est notamment lui qui informa Jean Marx dans une lettre écrite à Budapest le 31 mars 1929 de « la dernière phase de la manœuvre hongroise dans l'affaire de la chaire » :

Disposés aux concessions dernières, les Hongrois ont brusquement changé d'attitude ces jours-ci. Le revirement s'est produit au cours de la visite que M. Magyary vient de faire à Paris. À l'en croire, il aurait abouti à un accord de principe prévoyant 1) la fondation d'une chaire à la Sorbonne, 2) la désignation à ce poste de Tronchon. Seuls resteraient à régler les détails matériels.

Simultanément, je reçois de Meillet un mot où j'apprends que la Sorbonne « ne marche pas » et que « seule l'école reste ouverte ». [...] Dans ces conditions, je crois que le temps est venu d'accuser officiellement réception de la réponse hongroise de décembre dernier et de remettre les choses au point, si toutefois vous pensez toujours recourir à ce moyen, ainsi que vous me l'aviez fait entendre lors de nos conversations à Paris. 
Il ajoute encore que le ministre de France en Hongrie, « Monsieur de Vienne a été laissé par les gens d'ici dans l'ignorance de tout ce qu'ils trament. Seules des indiscrétions m'ont permis de le tenir au courant $»^{51}$.

Cette lettre signale déjà l'aboutissement du projet sans contribution financière hongroise et explique le fond de la lettre du ministre de France à Budapest citée en tête de l'article.

Un an plus tard, en février 1930, Sauvageot remet un rapport de huit pages dactylographiées dans lequel il dénonce la politique et le gouvernement hongrois ${ }^{52}$, les sociétés secrètes, la militarisation de la société et le danger que tout cela représente pour la paix dans la région ${ }^{53}$. Arrivé au ministère des Affaires étrangères, probablement par l'entremise de René Pinon, le document - semble-t-il - ne fait aucun effet. Si ce rapport est tout de même intéressant, c'est parce qu'il permet de mesurer l'état d'esprit et la vision que Sauvageot avait de la Hongrie de son temps l'année précédant son retour en France pour enseigner la langue et la culture hongroises.

On n'a d'ailleurs fort peu d'informations sur ses cours et le contenu de son enseignement. Ce sont plutôt ses publications qui peuvent nous renseigner.

En 1937, il fait une conférence à l'Institut de linguistique de l'Université de Paris sur La structure de la langue hongroise. L'image qu'il montre des Hongrois ne diffère pas de ce qu'il en avaient dit treize ans plus tôt dans Les Langues $d u$ monde. Ce qui caractérise la structure de la langue hongroise,

telle qu'elle nous apparaît aujourd'hui, c'est sa nature composite. Elle est constituée par un mélange de traits anciens et d'innovations. Les traits anciens procèdent du type archaïque que l'on est en droit de restituer pour les langues finno-ougriennes anciennes et les innovations semblent dues en partie à l'influence exercée sur le hongrois, durant des siècles, par les langues de type indo-européen.

La même année, après six ans d'expérience de l'enseignement du hongrois, il publie son livre intitulé La Découverte de la Hongrie où il déclare :

L'apprentissage du hongrois n'est pas facile. Non que le système grammatical soit compliqué. Au contraire, rien n'est plus claire qu'une forme hongroise. [...] Mais ce système est employé perpétuellement hors de son sens originel, pour exprimer une réalité européenne, avec des moyens forgés il y a plus que quinze cents ans sur les confins de l'Europe et de l'Asie par des ancêtres qui vivaient la grande civilisation de la steppe turque.

Le miracle de la langue hongroise, c'est d'être parvenue à réaliser ce prodige : exprimer toute l'Europe d'aujourd'hui. Il y a fallu un effort colossal qui a duré plus de mille ans et qui ne se relâche pas non plus aujourd'hui, un labeur de tous les instants, un travail perpétuel de création continue de la langue. [...] Ce qui caractérise donc au plus haut point le peuple hongrois, c'est sa faculté de s'adapter. ${ }^{54}$ 
Il n'est pas difficile d'identifier le cœur du problème : avant de retourner en France, pour Sauvageot, les études finno-ougriennes signifiaient la recherche des origines de cette famille de langues, et cette recherche a donné (ou a emprunté) une conception générale de l'histoire de la langue dominée par une interprétation idéologique et politique très restrictive. Or, à l'École des langues orientales, Sauvageot est titulaire de la chaire des langues finno-ougriennes où il est tenu d'assurer « l'enseignement théorique (grammaire descriptive) et une partie de l'enseignement pratique (explication de textes difficiles, exercices de traduction, correction des devoirs écrits, etc) $»^{55}$. Il doit donc renvoyer en arrière plan ses connaissances d'historien du langage et enseigner plutôt la langue vivante, telle qu'elle est utilisée. Pour ce faire, il s'appuie sur des exemples du langage courant, qu'il explique en français. Cette pratique lui apprend

\begin{abstract}
qu'une langue est un tout en soi. Elle est l'instrument par lequel s'est exprimée la pensée d'une collectivité, en l'occurrence une nation formée au cours de nombreux siècles d'histoire et non seulement de préhistoire. Il est impossible de dissocier la langue du contenu pensant qu'elle véhicule, c'est-à-dire de la séparer de la littérature à laquelle elle a donné naissance, pas plus que des institutions du peuple qui s'en sert. En d'autres termes, enseigner une langue, c'est enseigner en même temps les traits principaux d'une civilisation. Et alors les méthodes de la grammaire comparée n'ont plus cours. ${ }^{56}$
\end{abstract}

Ce texte de 1958 montre que la réflexion de Sauvageot s'est éloignée - très certainement sous l'influence de la pratique de l'enseignement, mais aussi en raison du changement de contexte politique et historique en Europe - de l'interprétation politique de la langue héritée d'Antoine Meillet. Nous avons vu plus haut qu'en dépit du peu de mérite qu'il accordait à la civilisation hongroise, Meillet a joué un rôle crucial dans l'institutionnalisation des études hongroises en France après la première guerre mondiale. Car, malgré ses convictions et prises de positions politiques, il n'a cessé de penser en linguiste et il a compris que la France avait besoin, quel que soit le contexte politique, de développer et préserver les connaissances sur une langue et une civilisation ayant survécu mille ans en Europe, loin de sa famille linguistique, au milieu de langues et civilisations indo-européennes étrangères. En la personne de son élève, les considérations contradictoires d'Antoine Meillet sur l'objet de ses études et recherches se sont perpétuées sans pour autant empêcher l'œuvre importante et utile qu'Aurélien Sauvageot a accompli, au cours des six décennies de sa carrière, pour la représentation de la culture hongroise en France. 


\section{Références}

A. G., « Magyar egyetemi tanár véleménye alapján avattak doktorrá a Sorbonne-on egy fiatal francia tudóst », Az Est, 18 juin 1929, p. 10.

Ablonczy Balázs, «A francia külpolitika magyarságképe a két világháború között », Limes, $\mathrm{n}^{\circ} 2$, 2008, p. 27-41. http://www.jamk.hu/ek/folyoirat/folyoirat/limes/2008_2.pdf

Bergounioux, Gabriel, « Entre épistémologie de la grammaire comparée et figure de l'intellectuel : la situation d'Antoine Meillet ", in Gabriel Bergounioux et Charles de Lamberterie, Meillet aujourd'hui, Leuven-Paris, Peeters, 2006, p. 109-136.

Hóman Bálint, « Les récentes études relatives à l'origine du peuple hongrois », Revue des Études hongroises et finno-ougriennes, avril-septembre 1924, p. 156-171.

Marès, Antoine, Louis Eisenmann et l'Europe centrale (1897-1937), in Regards sur l'indomptable Europe du centre-est du XVIII siècle à nos jours, textes réunis par Jerzy Kloczowski Daniel Beauvois, Yves-Marie Hilaire, Revue du Nord (hors série), nº 10, 1996, Lille, p. 223-242.

Meillet, Antoine, Les langues dans l'Europe nouvelle, Paris, Payot, 1918 [seconde édition : 1928].

Meillet, Antoine, «Les Langues et les nationalités », Scientia, vol. 18, 1915, https://fr.wikisource. org/wiki/Les_Langues_et les_nationalit\%C3\%A9s.

Meillet, Antoine, "Robert Gauthiot», in Annuaire 1917-1918. École pratique des hautes études, Section des sciences historiques et philologiques, Année 1917, vol. 50, nº 1, p. 57-61, http:// www.persee.fr/doc/ephe_0000-0001_1917_num_1_1_9344

Meillet, Antoine, "La Situation linguistique en Russie et en Autriche-Hongrie », Scientia, vol. 23, 1918, https://fr.wikisource.org/wiki/La_Situation_linguistique_en_Russie_et_en_AutricheHongrie

Sauvageot, Aurélien, " [sans titre] », in Vingt-cinq ans d'enseignement en France des langues finno-ougriennes, sous la dir. de Jean Gergely et Aimo Sakari, Paris, Institut-Hongrois de Paris, 1958, 9-19.

Sauvageot, Aurélien, Découverte de la Hongrie, Paris, Alcan, 1937.

Sauvageot, Aurélien, "Langues finno-ougriennes et langues samoyèdes ", in Les Langues $d u$ monde, sous la dir. d'Antoine Meillet et Marcel Cohen, Paris, Champion, 1924, p. 153-183.

Sauvageot, Aurélien, "L'origine du peuple hongrois ", Revue des Études hongroises et finno-ougriennes, avril-septembre 1924, p. 106-116, http://acta.bibl.u-szeged.hu/25258/1/etudes hongroises_002_106-116.pdf

Sauvageot, Aurélien, Souvenirs de ma vie hongroise, Budapest, Collège Eötvös József, ELTE Institut Français de Budapest, 2013, http://honlap.eotvos.elte.hu/wp-content/uploads/2016/02/ Magyarorszagi_eletetutam_francia.pdf

Swiggers, Pierre, « Antoine Meillet (1866-1936). De la grammaire comparée à la sociologie du langage », Anamnèse, Petite anthologie des auteurs oubliés, 2008, vol. 2, n ${ }^{\circ}$, p. 141-154.

Zichy, Étienne, «L'Origine du peuple hongrois », Revue des Études hongroises et finno-ougriennes, 1923, p. 5-14. http://acta.bibl.u-szeged.hu/39444/1/etudes_hongroises_001.pdf 


\section{Notes}

1 Lettre de Louis de Vienne, ministre de France en Hongrie à Aristide Briand, ministre des affaires étrangères, le 6 décembre 1929, ministère des Affaires étrangères, Archives diplomatiques (La Courneuve) (désormais: MAE AD), fonds du Service des oeuvres françaises à l'étranger (désormais: SOFE), carton $n^{\circ} 147$. Voir aussi Aurélien Sauvageot, Souvenirs de ma vie hongroise, Budapest, Collège Eötvös József, ELTE - Institut Français de Budapest, 2013, 327-328, http://honlap.eotvos.elte.hu/wp-content/uploads/2016/02/Magyarorszagi_eletetutam_francia. pdf [Dernier accès à toutes les sources internet citées dans cet article : le 31 août 2019.] Mes recherches dans les archives françaises ont été subventionnées par le Programme Klebelsberg de l'Institut Balassi (Budapest, Hongrie).

2 Les langues finno-ougriennes sont, en Europe, le hongrois, le finnois et l'estonien, auxquelles s'ajoutent, en Asie, quelques autres langues ayant moins de locuteurs.

3 Dossier intitulé « Conférences et cours complémentaires : cours libres et gratuits, 1884-1933», Archives nationales (Paris) (désormais : AN), fonds de l'École des langues orientales vivantes, F/17/13611. Sur Eisenmann, voir Antoine Marès : Louis Eisenmann et l'Europe centrale (18971937), in Regards sur l'indomptable Europe du centre-est du XVIIIe siècle à nos jours, textes réunis par Jerzy Kloczowski, Daniel Beauvois, Yves-Marie Hilaire, Revue du Nord (hors série), no 10, 1996, Lille, 223-242.

4 Lettre du ministre de l'Instruction publique et des Beaux-Arts à Paul Deschanel, président de la Chambre de Députés, le 3 juillet 1912, dossier intitulé Conférences et cours complémentaires : cours libres et gratuits, 1884-1933, AN, Fonds de l'École des langues orientales vivantes, $\mathrm{F} / 17 / 13611$.

5 « Aide-mémoire pour l'organisation des rapports intellectuels franco-hongrois », rédigé par Louis Eisenmann, MAE AD, SOFE, carton $n^{\circ} 147$.

6 Société de linguistique de Paris, Antoine MEILLET, http://www.slp-paris.com/spip.php?article4

7 Ibid.

8 Pierre Swiggers, «Antoine Meillet (1866-1936). De la grammaire comparée à la sociologie du langage », Anamnèse, Petite anthologie des auteurs oubliés, 2008, vol. 2, n 0, p. 144.

9 Avant-propos de la première édition de l'ouvrage d'Antoine Meillet, Les langues dans l'Europe nouvelle (Paris, Payot, 1918, p. 7) ; repris dans la seconde édition avec un changement de temps : le verbe de la dernière proposition change du présent à l'imparfait (1928, p. VIII).

10 Dans son journal rédigé entre 1896 et 1906, Meillet cite parmi ses objectifs « [d']organiser institutionnellement la linguistique française pour en faire la référence mondiale dans le domaine (face à l'Allemagne)». Gabriel Bergounioux : «Entre épistémologie de la grammaire comparée et figure de l'intellectuel : la situation d'Antoine Meillet », in Gabriel Bergounioux et Charles de Lamberterie, Meillet aujourd'hui, Leuven-Paris, Peeters, 2006, p. 118.

11 Antoine Meillet, «Robert Gauthiot», in Annuaire 1917-1918. École pratique des hautes études, Section des sciences historiques et philologiques, Année 1917, vol. 50, nº 1, p. 59-60, http:// www.persee.fr/doc/ephe_0000-0001_1917_num_1_1_9344

12 Sauvageot, Souvenirs, p. 14.

13 Ibid., p. 14-15.

14 Ibid., p. 15.

15 Ibid.

16 « État des services d'Aurélien Sauvageot du 31 décembre 1936 », AN, fonds de l'INALCO, carton $n^{\circ} 20100053 / 22$ de la Direction des ressources humaines, dossier personnel d'Aurélien Sauvageot. 
17 «État des services d'Aurélien Sauvageot du 1er janvier 1933 » et « État civil de M. Sauvageot », le 15 février 1942, AN, fonds de l'INALCO, carton n² 20100053/22 de la Direction des ressources humaines, dossier personnel d'Aurélien Sauvageot.

18 «État civil de Sauvageot » du 15 février 1942 dit : « Classé 6ème, n’est pas admis ! (ibid.).

19 Sauvageot, Souvenirs, p. 115.

20 Ibid., p. 329.

21 Lettre d'Aurélien Sauvageot à Géza Bartoniek, directeur du Collège Eötvös, le 29 octobre 1923, Archives du Collège Eötvös, MDKL 1/f, carton 46, dossier 84/a. (Je remercie le directeur actuel du Collège Eötvös, László Horváth de l'autorisation de publier cette lettre et de m'avoir communiqué les fonds du Collège concernant mon sujet. Mes remerciements vont également à Attila Boda, archiviste du Collège pour son aide dans mes recherches.)

22 Étienne Zichy, "L'Origine du peuple hongrois », Revue des Études hongroises et finno-ougriennes, 1923, p. 5-14. http://acta.bibl.u-szeged.hu/39444/1/etudes_hongroises_001.pdf

23 Aurélien Sauvageot, "L'origine du peuple hongrois ", Revue des Études hongroises et finno-ougriennes, avril-septembre 1924, p. 106, http://acta.bibl.u-szeged.hu/25258/1/etudes_hongroises_002_106-116.pdf

24 Voir le résumé de la discussion en français dans le même numéro de la revue : Bálint Hóman, « Les récentes études relatives à l'origine du peuple hongrois », Revue des Études hongroises et finno-ougriennes, avril-septembre 1924, p. 156-171.

25 Sauvageot, L'origine du peuple hongrois, p. 107.

26 Ibid., p. 113.

27 Lettre de Zoltán Gombocz à Antoine Meillet, le 25 mai 1924, MAE AD, SOFE, carton nº 149.

28 « Je me méfie de son imagination »-écrit Meillet de Sauvageot dans une lettre adressée au ministère des Affaires étrangères (probablement à Jean Marx) le 6 juillet 1924 (MAE AD, SOFE, carton $n^{\circ} 149$ ), un sentiment que le chercheur qui compare des documents d'archives aux Souvenirs de Sauvageot ne peut que partager.

29 Suite à un conflit avec Louis Eisenmann et au conseil de Meillet, Sauvageot change de sujet secondaire. Faute de documents originaux sur l'affaire, seuls les souvenirs de Sauvageot peuvent nous en donner une idée. Voir Sauvageot, Souvenirs, p. 146-150.

30 «Lettres de Sauvageot à ses amis », Fonds Sauvageot, Bibliothèque universitaire des Fenouillères, Université Aix-Marseille, Aix-en-Provence.

31 Swiggers, Antoine Meillet, p. 144.

32 Aurélien Sauvageot, "Langues finno-ougriennes et langues samoyèdes », in Les Langues du monde, sous la dir. d'Antoine Meillet et Marcel Cohen, Paris, Champion, 1924, p. 176.

33 Sauvageot, Langues finno-ougriennes, p. 182.

34 Meillet, Les langues dans l'Europe nouvelle, 1918, p. 10-11 ; 1928, p. 2-3.

35 Antoine Meillet, «Les Langues et les nationalités », Scientia, vol. 18, 1915, https://fr.wikisource.org/wiki/Les_Langues_et_les_nationalit\%C $3 \%$ A9s.

36 Antoine Meillet, "La Situation linguistique en Russie et en Autriche-Hongrie », Scientia, vol. 23, 1918, https://fr.wikisource.org/wiki/La_Situation_linguistique_en_Russie_et_en_Autriche-Hongrie

37 Ibid.

38 Meillet, Les langues dans l'Europe nouvelle, 1918, p. 236.

39 Ibid. , p. 208.

40 Thèse principale : Recherches sur le vocabulaire des langues ouralo-altaïques, thèse secondaire : L'emploi de l'article en gothique.

41 A. G., « Magyar egyetemi tanár véleménye alapján avattak doktorrá a Sorbonne-on egy fiatal francia tudóst », Az Est, 18 juin 1929, p. 10. 
42 Lettre de l'administrateur de l'Ecole des langues orientales [Paul Boyer] au Ministre de l'Instruction publique et des Beaux-Arts, le 18 août 1931, AN, fonds de l'INALCO, carton $\mathrm{n}^{\circ}$ 20100053/22 de la Direction des ressources humaines, dossier personnel d'Aurélien Sauvageot.

43 Lettre de Louis de Robien, chargé d'affaires de France à Budapest à Aristide Briand, ministre des Affaires étrangères, le 8 juin 1925, MAE AD, SOFE, carton $n^{\circ} 147$.

44 Lettre d'Aurélien Sauvageot à Archicube, le 14 mai 1925, MAE AD, SOFE, carton n 147. À l'heure actuelle de mes recherches, je ne peux pas affirmer avec certitude qui est le destinataire de la lettre. Les Souvenirs de Sauvageot donnent une piste qui reste à vérifier : il raconte l'histoire de sa première rencontre avec Louis Eisenmann qui se présente à lui comme « archicube Eisenmann » (p. 118).

45 Lettre de Paul Boyer au SOFE (probablement à Paul-Emile Naggiar), le 28 mai 1925, MAE $\mathrm{AD}$, SOFE, carton $\mathrm{n}^{\circ} 147$.

46 Lettre du ministre des Affaires étrangères au ministre de l'Instruction publique, le 17 juin 1925 , MAE AD, SOFE, carton $n^{\circ} 147$.

47 Meillet rend compte de son entrevue avec Sauvageot dans une lettre manuscrite (et difficilement lisible) adressée à Paul Boyer le 29 mai 1925 : «J'ai revu Sauvageot mercredi. Je lui ai parlé finement, et lui ai dit que rien ne peut être fait pour lui tant qu'il n'est pas prouvé, par un travail solide et original, qu'il est qualifié. Il est sorti manifestement abattu. Mais il fallait lui montrer la réalité. En aucun cas, il ne faut le décourager. Il a de l'invention et la qualité de linguiste. Si son caractère le $[\ldots \ldots \ldots . . .$. . Mais il faut lui donner toutes les possibilités d'[...]. Nous n'avons pas assez d'hommes pour le sacrifier." AN, fonds de l'INALCO, carton n ${ }^{\circ}$ 20100053/22 de la Direction des ressources humaines, dossier personnel d'Aurélien Sauvageot.

48 Lettre de Jean Marx (Section des écoles, Service des oeuvres françaises) à Aurélien Sauvageot, le 10 septembre 1925, MAE AD, SOFE, carton $\mathrm{n}^{\circ} 149$.

49 Sauvageot, Souvenirs, p. 124-126.

50 Ibid., p. 279-281.

51 Lettre d'Aurélien Sauvageot au Service des œuvres françaises, le 31 mars 1929, MAE AD, SOFE, carton $\mathrm{n}^{\circ} 149$.

52 Il écrit entre autres du gouvernement qu'il « n'a pas oublié qu'il a failli tomber en 1926 à cause de l'intervention énergique de M. Clinchant [ministre de France en Hongrie de l'époque]. Sa haine à l'égard de la France s'en trouve exaspérée. Aussi saisit-il toutes les occasions possibles de nuire au prestige et aux intérêts de notre pays. » Rapport d'Aurélien Sauvageot, MAE AD, Europe 1918-1940, Hongrie, vol. 144, ff. 1-8.

53 Balázs Ablonczy, «A francia külpolitika magyarságképe a két világháború között », Limes, $\mathrm{n}^{\circ}$ 2, 2008, p. 34. http://www.jamk.hu/ek/folyoirat/folyoirat/limes/2008_2.pdf

54 Aurélien Sauvageot, Découverte de la Hongrie, Paris, Alcan, 1937, p. 17.

55 Rapport sur dix ans d'activité du cours des langues finno-ougriennes, [1941], AN, fonds de l'INALCO, carton n ${ }^{\circ} 20100053 / 22$ de la Direction des ressources humaines, dossier personnel d'Aurélien Sauvageot.

56 Article d'Aurélien Sauvageot paru sans titre dans Vingt-cinq ans d'enseignement en France des langues finno-ougriennes, sous la dir. de Jean Gergely et Aimo Sakari, Paris, Institut-Hongrois de Paris, 1958, 11-12.

Open Access. This is an open-access article distributed under the terms of the Creative Commons Attribution 4.0 International License (https://creativecommons.org/licenses/by/4.0), which permits unrestricted use, distribution, and reproduction in any medium, provided the original author and source are credited, a link to the CC License is provided, and changes - if any - are indicated. (SID_1) 
